\title{
Properties of $N^{*}(1535)$ at Finite Density in the Extended Parity-Doublet Models
}

\author{
Hungchong Kim *, D. Jido ${ }^{\dagger}$ and M. Oka ${ }^{\ddagger}$ \\ Department of Physics, Tokyo Institute of Technology, Meguro, Tokyo 152-8551 Japan
}

\begin{abstract}
We improve so called "naive" and "mirror" models for the positive and negative parity nucleons, $N$ and $N^{*}$, by introducing nonlinear terms allowed by chiral symmetry. Both models in this improvement reproduce the observed nucleon axial charge in free space and reveal interesting density dependence of the axial charges for $N$ and $N^{*}$, and the doublet masses. A remarkable difference between the two models is found in the off-diagonal axial charge, $g_{A N N^{*}}$, which could appear either as suppression or as enhancement of $N^{*} \rightarrow \pi N$ decay in the medium.
\end{abstract}

PACS numbers: 11.30.Rd,12.39.Fe,14.20.Gk,21.65.+f

Key Words : chiral symmetry, negative parity nucleon, finite density

\footnotetext{
*Email : hung@th.phys.titech.ac.jp

†Email : jido@th.phys.titech.ac.jp

${ }^{\ddagger}$ Email : oka@th.phys.titech.ac.jp
} 


\section{INTRODUCTION}

The negative-parity nucleon resonance, $N^{*}(1535)$, is believed to play a dominant role in $\eta$ productions via electromagnetic or hadronic probes [1]. There have been some attempts to understand $N^{*}(1535)$ resonance as well as its decaying properties based on effective models [2]. More recently, the resonance has been studied in the framework of QCD sum rules by using the interpolating field either with a covariant derivative [3] or without [4].

One interesting attempt in the study of $N^{*}$ is to construct a chiral model where the resonance is regarded as a chiral partner of a nucleon. As chiral symmetry and its spontaneous breaking is important in describing low energy nuclear physics, it seems natural to extend the baryonic sector of chiral Lagrangian and include the negative-parity baryon.

Indeed, such extension was proposed by DeTar and Kunihiro [5]. In their model, the negative-parity baryon, under the chiral rotation, is allowed to transform in the opposite direction of the way that the positive-parity baryon transforms. In this assignment of the chiral transformation which we call "mirror assignment", the nucleon may have a nonzero mass, $m_{0}$, even when the chiral symmetry is restored. This seems to support the results from lattice calculations at finite temperature [6]. One interesting prediction of this model is that the axial charge of $N^{*}$ has the opposite sign of the nucleon axial charge, which seems to agree with the QCD sum rule approach proposed by Lee and Kim [3].

On the other hand, as pointed out by Jido et al. [7], one can also construct a chiral model by allowing the negative-parity baryon to transform in the same way as the positive-parity baryon under the chiral rotation. Under this assignment which we call "naive assignment", the $\pi N N^{*}$ coupling is zero and the $N^{*}$ axial charge has the same sign as the nucleon. The vanishing $\pi N N^{*}$ coupling is at least qualitatively consistent with the suppression of the coupling observed in experiment. This way of including the negative-parity baryon leads to two independent linear sigma models and the masses of $N$ and $N^{*}$ vanish as the chiral symmetry is restored. This model yields the results consistent with the QCD sum rule approach proposed by Jido et al. [4].

At present, a question remains as to which assignment of chiral symmetry is realized in the real world. Finding the answer will help us not only to understand $N^{*}$ itself but also to make reliable predictions on $N^{*}$ properties as chiral symmetry is restored. Certainly the relative sign of the $N^{*}$ axial charge, if measured, can be used to determine the realistic model. But it will be difficult to measure the axial charge because $N^{*}$ decays strongly to $\eta N$ or $\pi N$. Instead, it will be useful to pursue other perspectives of the two models.

To do so, we propose in this work a minimal extension of each model by introducing additional terms in the Lagrangian allowed by respective chiral symmetry. Similar extension of the original linear sigma model, normally known as modified linear sigma model, has been proposed in order to have the axial charge of nucleon greater than unity [8]. Such extension will be interesting in our nucleon doublet models as it may provide different predictions for the doublet masses and their couplings to pion as the chiral symmetry is restored, which then may be measured in pion photoproductions in nuclei or possibly in heavy-ion collisions.

Our paper is organized as follows. In Section [1], we propose an extension of the naive model after briefly reviewing the original naive model. We discuss why the original naive model needs to be improved and make a nonlinear transformation of the extended model to describe $\pi$ interactions in the pseudovector coupling scheme. In Section III, we provide a 
similar extension for the mirror model. In Section एV, we apply our models to a system with finite baryon density and see how the doublet masses and the axial charges are changed as respective chiral symmetry is being restored. Throughout this paper, $N^{*}$ denotes $N(1535)$.

\section{EXTENSION OF THE NAIVE MODEL}

The well-known linear sigma model of Gell-Mann and Lévy can be constructed by requiring its Lagrangian to be invariant under the following chiral transformations,

$$
N_{1} \rightarrow N_{1}-i \boldsymbol{\alpha} \cdot \frac{\boldsymbol{\tau}}{2} \gamma_{5} N_{1} ; \quad \boldsymbol{\pi} \rightarrow \boldsymbol{\pi}+\boldsymbol{\alpha} \sigma ; \quad \sigma \rightarrow \sigma-\boldsymbol{\pi} \cdot \boldsymbol{\alpha} .
$$

Here $\boldsymbol{\alpha}$ denotes an infinitesimal vector for the transformations and $\boldsymbol{\tau}$ refers to the Pauli matrices acting on the isospin space. Of course, one has to impose the symmetry under the vector transformation in constructing the Lagrangian. One possible way to include the negative-parity baryon, $N_{2}$, in the linear sigma model is to construct a Lagrangian invariant under the further transformation of

$$
N_{2} \rightarrow N_{2}-i \boldsymbol{\alpha} \cdot \frac{\boldsymbol{\tau}}{2} \gamma_{5} N_{2}
$$

One can construct a simple Lagrangian invariant under these chiral transformations [7],

$$
\begin{aligned}
\mathcal{L}_{N}^{0}= & \sum_{j=1,2}\left[\bar{N}_{j} i \not \partial N_{j}-a_{j} \bar{N}_{j}\left(\sigma+i \gamma_{5} \boldsymbol{\tau} \cdot \boldsymbol{\pi}\right) N_{j}\right] \\
& -a_{3}\left[\bar{N}_{1}\left(\gamma_{5} \sigma+i \boldsymbol{\tau} \cdot \boldsymbol{\pi}\right) N_{2}-\bar{N}_{2}\left(\gamma_{5} \sigma+i \boldsymbol{\tau} \cdot \boldsymbol{\pi}\right) N_{1}\right]+\mathcal{L}_{m} .
\end{aligned}
$$

Here we do not specify the mesonic part $\mathcal{L}_{m}$ because it is not needed in most discussions below. This model is called as "naive" because the Lagrangian is constructed by the naive extension of chiral symmetry to the negative-parity baryon given in Eq. (2).

Normally, a nucleon acquires its mass by spontaneous breaking of chiral symmetry. In this case with the additional baryonic field, we have a mass matrix with nonzero off-diagonal

elements (namely the term proportional to $a_{3}$ ) in the baryon doublet basis $\left(\begin{array}{l}N_{1} \\ N_{2}\end{array}\right)$. The physical masses for $N$ and $N^{*}$ can be obtained by diagonalizing the mass matrix. However, under the diagonalization, the matrix representing the interactions between pion and baryon doublets is also diagonalized, which therefore leads to the coupling, $g_{\pi N N^{*}}=0$. This implies that this model is nothing but the sum of two independent sigma models. Furthermore, using the physical values of the doublet masses and the pion decay constant $f_{\pi}$, we find $g_{\pi N N}=10$, which is about $30 \%$ smaller than what is normally known. This discrepancy is because the axial charges for both $N$ and $N^{*}$ are unity independent of the parameters $a_{i}$ or $\langle\sigma\rangle$. Since, within this model, no other adjustments are allowed to achieve consistency with phenomenology in the tree level, this naive model needs to be improved.

The meson-baryon couplings of the Lagrangian Eq. (31) are linear in the meson field. One natural extension of the naive model is to add quadratic meson-baryon interactions to $\mathcal{L}_{N}^{0}$,

$$
\begin{aligned}
\mathcal{L}_{N}^{1}= & \sum_{j=1,2} b_{j} \bar{N}_{j}\left[\left(\frac{\boldsymbol{\tau}}{2} \cdot \boldsymbol{\pi} \not \partial \sigma-\sigma \frac{\boldsymbol{\tau}}{2} \cdot \not \partial \boldsymbol{\pi}\right) \gamma_{5}-\frac{\boldsymbol{\tau}}{2} \cdot(\boldsymbol{\pi} \times \not \partial \boldsymbol{\pi})\right] N_{j} \\
& +b_{3}\left\{\bar{N}_{1}\left[\left(\frac{\boldsymbol{\tau}}{2} \cdot \boldsymbol{\pi} \not \partial \sigma-\sigma \frac{\boldsymbol{\tau}}{2} \cdot \not \partial \boldsymbol{\pi}\right)-\frac{\boldsymbol{\tau}}{2} \cdot(\boldsymbol{\pi} \times \not \partial \boldsymbol{\pi}) \gamma_{5}\right] N_{2}+\text { h.c. }\right\} .
\end{aligned}
$$


It is easy to see that these terms, whose form being vector-vector plus axial-vector-axialvector, are invariant under the chiral transformations, Eqs. (11) and (2). Note that these are constructed by using the least number of meson fields and derivatives among all possibilities allowed by the naive assignment of chiral symmetry. Chiral symmetry does not prevent from having other terms containing more meson fields or more derivatives. In this sense, our extension should be regarded as minimal. The truncation in number of mesons and derivatives makes sense when contributions from the additional terms are not so large. Similar modification of the linear sigma model has been proposed by Lee [9] and later used by Akhmedov [8] in an effort to have the axial charge greater than 1 in the tree level. In our case, because of the negative-parity baryon, we have the off-diagonal terms in addition. As the nucleon mass matrix is unchanged by including $\mathcal{L}_{N}^{1}$, the previous mass eigenstates still form the physical basis in this extension.

However, in this extension, the pion couples to the nucleons both in pseudoscalar type and in pseudovector type. It is well known [10] that the pseudoscalar coupling scheme leads to too large $s$-wave $\pi N$ scattering length from the Born terms, which then needs to be reduced by the $t$-channel sigma exchange between the pion and the nucleon. This sensitive cancellation however is not necessary when one uses the pseudovector coupling scheme obtained from nonlinear transformations of the linear sigma model. Furthermore, a Hartree-Fock calculation of nuclear matter shows [11] that the pseudoscalar coupling scheme is problematic in describing normal nuclear matter. Therefore, it is favorable to have a model in the pseudovector coupling scheme only. This can be achieved by the nonlinear realization of chiral symmetry, as originally studied by Weinberg [12].

Motivated by Weinberg, we take the following nonlinear transformations,

$$
\begin{aligned}
\psi_{j} & =\frac{1}{\sqrt{1+\left(\frac{\pi^{\prime}}{2 f_{\pi}}\right)^{2}}}\left[1-\frac{i}{2 f_{\pi}} \boldsymbol{\pi}^{\prime} \cdot \boldsymbol{\tau} \gamma_{5}\right] N_{j} \text { where } j=1,2, \\
\frac{\boldsymbol{\pi}^{\prime}}{2 f_{\pi}} & =\frac{\boldsymbol{\pi}}{\sqrt{\sigma^{2}+\pi^{2}}+\sigma} ; \quad \sigma^{\prime}=\sqrt{\sigma^{2}+\pi^{2}} .
\end{aligned}
$$

We want to stress that the negative-parity baryon is transformed similarly to the positiveparity baryon so that the naive assignment of chiral symmetry is realized nonlinearly. After applying the nonlinear transformations to the Lagrangian $\left(\mathcal{L}_{N}^{0}+\mathcal{L}_{N}^{1}\right)$ and implementing the spontaneous symmetry breaking, we obtain

$$
\begin{aligned}
\mathcal{L}_{N}^{N L}= & \bar{\psi} i \not \partial \psi+\frac{1}{1+\left(\frac{\pi^{\prime}}{2 f_{\pi}}\right)^{2}} \bar{\psi} \not \partial \boldsymbol{\pi}^{\prime} \cdot \boldsymbol{\tau} \gamma_{5} \boldsymbol{\Pi} \psi-\frac{\left(1 / 2 f_{\pi}\right)^{2}}{1+\left(\frac{\pi^{\prime}}{2 f_{\pi}}\right)^{2}} \bar{\psi} \boldsymbol{\tau} \cdot\left(\boldsymbol{\pi}^{\prime} \times \not \partial \boldsymbol{\pi}^{\prime}\right) \psi \\
& -\bar{\psi} \mathbf{M} \psi+\mathcal{L}_{m}
\end{aligned}
$$

where we have introduced the column vector, $\psi \equiv\left(\begin{array}{l}\psi_{1} \\ \psi_{2}\end{array}\right)$. In this basis, the pion coupling and the baryon mass matrices are defined respectively as

$$
\boldsymbol{\Pi}=\frac{1}{2 f_{\pi}}\left(\begin{array}{cc}
1-b_{1} \sigma_{0}^{2} & -b_{3} \sigma_{0}^{2} \gamma_{5} \\
-b_{3} \sigma_{0}^{2} \gamma_{5} & 1-b_{2} \sigma_{0}^{2}
\end{array}\right) ; \quad \mathbf{M}=\sigma_{0}\left(\begin{array}{cc}
a_{1} & a_{3} \gamma_{5} \\
-a_{3} \gamma_{5} & a_{2}
\end{array}\right)
$$

where $\sigma_{0}$ denotes the expectation value of $\sigma^{\prime}$ whose value in vacuum is the pion decay constant $f_{\pi}$, and the fluctuation of $\sigma^{\prime}$ field around $\sigma_{0}$ is neglected. Note that the nonlinear 
transformations eliminate the pseudoscalar coupling of the pion. The $\sigma_{0}$ dependence of the couplings is now explicit, while nonlinear sigma models constructed without facilitating the linear sigma model lead to no explicit $\sigma_{0}$ dependence of the couplings and therefore we may not be able to study phenomena related to chiral symmetry restoration.

Now, because of the new terms involving two mesons, the pion-nucleon coupling matrix is different from the mass matrix. Diagonalization of the mass matrix does not simultaneously diagonalize the coupling matrix. Therefore, the $\pi N N^{*}$ coupling picks up a nonzero value in the tree level, which is certainly consistent with experimental facts. Moreover $\sigma_{0}$ is just an overall factor in the mass matrix. Thus the unitary matrix which diagonalizes the mass matrix does not depend on $\sigma_{0}$. It implies that, after the diagonalization, the coupling matrix will take the form,

$$
\Pi \rightarrow \frac{1}{2 f_{\pi}}\left(\begin{array}{cc}
1+B_{1} \sigma_{0}^{2} & B_{2} \sigma_{0}^{2} \gamma_{5} \\
B_{2} \sigma_{0}^{2} \gamma_{5} & 1+B_{3} \sigma_{0}^{2}
\end{array}\right)
$$

with some constants, $B_{i}(i=1,2,3)$. The matrix for the axial charges can be simply read off, giving

$$
\left(\begin{array}{cc}
g_{A N N} & g_{A N N^{*}} \\
g_{A N N^{*}} & g_{A N^{*} N^{*}}
\end{array}\right) \equiv\left(\begin{array}{cc}
1+B_{1} \sigma_{0}^{2} & B_{2} \sigma_{0}^{2} \\
B_{2} \sigma_{0}^{2} & 1+B_{3} \sigma_{0}^{2}
\end{array}\right)
$$

The constants, $B_{1}$ and $B_{2}$, can be determined by fitting experimental values for $\pi N N$ and $\pi N N^{*}$ couplings respectively. Specifically, the partial decay width for $N^{*} \rightarrow \pi N$ can be evaluated straightforwardly from the $\pi N$ self-energy of $N^{*}$,

$$
\Gamma_{\pi N}=\frac{3 f_{\pi N N^{*}}^{2}}{8 \pi M_{-}^{2}}|\mathbf{k}|\left(M_{-}-M_{+}\right)\left[\left(M_{-}+M_{+}\right)\left(M_{-}^{2}-M_{+}^{2}-m_{\pi}^{2}\right)+2 M_{+} m_{\pi}^{2}\right]
$$

where $|\mathbf{k}|$ is the momentum carried by the emitted pion and $f_{\pi N N^{*}} \equiv B_{2} \sigma_{0}^{2} / 2 f_{\pi}$ is the offdiagonal pseudovector coupling. From $\Gamma_{\pi N}^{e x p} \sim 70 \mathrm{MeV}$, we obtain $f_{\pi N N^{*}}=1.17 \mathrm{GeV}^{-1}$, which then yields $B_{2}=25.6 \mathrm{GeV}^{-2}$ when the vacuum expectation value, $\sigma_{0}=93 \mathrm{MeV}$, is used. Also from the diagonal pseudovector coupling $f_{\pi N N} \equiv\left(1+B_{1} \sigma_{0}^{2}\right) / 2 f_{\pi}=6.77 \mathrm{GeV}^{-1}$, we find $B_{1}=30.06 \mathrm{GeV}^{-2}$. Note that the small deviation of $g_{A N N}$ from unity and the suppressed off-diagonal coupling $f_{\pi N N^{*}}$ make $B_{1} \sigma_{0}^{2}=0.26$ and $B_{2} \sigma_{0}^{2}=0.217$, much smaller than unity, justifying the truncation in number of meson fields in Eqs. (3) and (田).

The masses of $N$ and $N^{*}$ are given as

$$
M_{+}=A_{1} \sigma_{0} ; \quad M_{-}=A_{2} \sigma_{0},
$$

where the constants, $A_{1}$ and $A_{2}$, are determined from the physical masses. When $\sigma_{0}$ is changed in the medium, $M_{ \pm}$will be changed linearly with $\sigma_{0}$. A similar scaling is proposed by Brown and Rho [13] where all hadron masses except for the Goldstone bosons are claimed to be scaled with the pion decay constant in the nuclear medium.

\section{EXTENSION OF THE MIRROR MODEL}

Another way of constructing the nucleon parity doublet model is to introduce a Lagrangian invariant under the "mirror assignment" of chiral symmetry [14], originally proposed by Lee [9] and later developed by DeTar and Kunihiro(DK) [5]. Under the mirror 
assignment, the negative-parity baryon is allowed to transform in the opposite direction to the way that the positive-parity baryon is transformed, namely

$$
\begin{aligned}
N_{1} & \rightarrow N_{1}-i \boldsymbol{\alpha} \cdot \frac{\boldsymbol{\tau}}{2} \gamma_{5} N_{1} ; \quad N_{2} \rightarrow N_{2}+i \boldsymbol{\alpha} \cdot \frac{\boldsymbol{\tau}}{2} \gamma_{5} N_{2}, \\
\boldsymbol{\pi} & \rightarrow \boldsymbol{\pi}+\boldsymbol{\alpha} \sigma ; \quad \sigma \rightarrow \sigma-\boldsymbol{\pi} \cdot \boldsymbol{\alpha} .
\end{aligned}
$$

Lagrangian invariant under this transformation is

$$
\begin{aligned}
\mathcal{L}_{M}^{0}= & \bar{N}_{d} i \not \partial N_{d}-g_{1} \bar{N}_{d}\left(\sigma+i \boldsymbol{\pi} \cdot \boldsymbol{\tau} \gamma_{5} \rho_{3}\right) N_{d}+g_{2} \bar{N}_{d}\left(\sigma \rho_{3}+i \boldsymbol{\pi} \cdot \boldsymbol{\tau} \gamma_{5}\right) N_{d} \\
& -i m_{0} \bar{N}_{d} \rho_{2} \gamma_{5} N_{d}+\mathcal{L}_{m}
\end{aligned}
$$

where we have introduced the parity doublet $N_{d} \equiv\left(\begin{array}{l}N_{1} \\ N_{2}\end{array}\right)$ for notational simplicity, and the Pauli matrices $\rho_{i}(i=1,2,3)$ which act on the nucleon parity doublets. Note that because of the mirror assignment, the Lagrangian is allowed to have the term containing $m_{0}$ which constitutes the off-diagonal element of the mass matrix.

In this case, unlike the naive case, the mass and the coupling matrices are not simultaneously diagonalized and we can have a nonzero value for $g_{\pi N N^{*}}$ without further introducing nonlinear terms. Using the experimental value for $g_{\pi N N^{*}}, M_{+}$and $M_{-}$, DK found,

$$
g_{1}=13 ; \quad g_{2}=3.2 ; \quad m_{0}=0.27 \mathrm{GeV} .
$$

This model is interesting because, in the restored phase of chiral symmetry, the nucleons still have the nonzero mass, $m_{0}$. The nonzero mass in the restored phase, though it is small, seems to be consistent with lattice calculations at finite temperature [6]. In addition, this approach reveals interesting behavior of $g_{A N N}$ as a function of $\sigma_{0}$. However, this model predicts $g_{\pi N N}=9.8$. The well-known value of $g_{\pi N N}$ can not be incorporated because the predicted axial charges for $N$ and $N^{*}$ are always less than 1 at the tree level. Therefore, the mirror model in the current form is somewhat too restrictive and needs to be improved.

One way to improve the mirror model is to take similar steps as in the previous section. Under the mirror assignment of chiral symmetry, the simplest extension of the model is to include the followings,

$$
\begin{aligned}
\mathcal{L}_{M}^{1}= & g_{3} \bar{N}_{1}\left(i \not \partial \sigma \gamma_{5}+\boldsymbol{\tau} \cdot \not \partial \boldsymbol{\pi}\right) N_{2}+h . c . \\
& +g_{4} \bar{N}_{1}\left[\left(\frac{\boldsymbol{\tau}}{2} \cdot \boldsymbol{\pi} \not \partial \sigma-\sigma \frac{\boldsymbol{\tau}}{2} \cdot \not \partial \boldsymbol{\pi}\right) \gamma_{5}-\frac{\boldsymbol{\tau}}{2} \cdot(\boldsymbol{\pi} \times \not \partial \boldsymbol{\pi})\right] N_{1} \\
& +g_{5} \bar{N}_{2}\left[-\left(\frac{\boldsymbol{\tau}}{2} \cdot \boldsymbol{\pi} \not \partial \sigma-\sigma \frac{\boldsymbol{\tau}}{2} \cdot \not \partial \boldsymbol{\pi}\right) \gamma_{5}-\frac{\boldsymbol{\tau}}{2} \cdot(\boldsymbol{\pi} \times \not \partial \boldsymbol{\pi})\right] N_{2} .
\end{aligned}
$$

Here the second and third lines contain two mesons similarly to Eq. (41). Note the sign of the axial-vector coupling term in the third line is opposite to that in the second line. This is because of the mirror assignment of chiral symmetry for the negative-parity baryon. The terms containing one meson are new and these are not allowed in the naive assignment. Of course, under chiral symmetry, terms with more mesons or more derivatives are also allowed. Therefore, this extension as in the naive case should be regarded as minimal. As before, the truncation in number of mesons and derivatives can be justified if the contributions of these corrections are not so large. 
Again, in this extension, the pion couples to the nucleons both in pseudoscalar type and in pseudovector type. Nonlinear transformations are required to eliminate the pseudoscalar coupling in favor of the pseudovector coupling. The mirror assignment of chiral symmetry is realized with slightly different nonlinear transformation for the parity doublets,

$$
\psi=\frac{1}{\sqrt{1+\left(\frac{\pi^{\prime}}{2 f_{\pi}}\right)^{2}}}\left[1-\rho_{3} \frac{i}{2 f_{\pi}} \boldsymbol{\pi}^{\prime} \cdot \boldsymbol{\tau} \gamma_{5}\right] N_{d},
$$

while $\pi$ and $\sigma$ are transformed as before. Note that $\rho_{3}$ indicates the sign difference for the negative-parity baryon. The nonlinear transformations and subsequent spontaneous symmetry breaking takes the Lagrangian $\left(\mathcal{L}_{M}^{0}+\mathcal{L}_{M}^{1}\right)$ into the same form as in Eq. (6) but the pion coupling and mass matrices now take different forms,

$$
\boldsymbol{\Pi}=\frac{1}{2 f_{\pi}}\left(\begin{array}{cc}
1-g_{4} \sigma_{0}^{2} & 2 g_{3} \sigma_{0} \gamma_{5} \\
2 g_{3} \sigma_{0} \gamma_{5} & -1-g_{5} \sigma_{0}^{2}
\end{array}\right) ; \quad \mathbf{M}=\left(\begin{array}{cc}
\left(g_{1}-g_{2}\right) \sigma_{0} & m_{0} \gamma_{5} \\
-m_{0} \gamma_{5} & \left(g_{1}+g_{2}\right) \sigma_{0}
\end{array}\right) .
$$

Since there is no dependence on $g_{3}, g_{4}$ and $g_{5}$ in the mass matrix, the mass eigenstates are the same as before the extension. The mass matrix is diagonalized by the unitary matrix

$$
\mathbf{U}=\frac{1}{2 \cosh \delta}\left(\begin{array}{cc}
e^{\frac{\delta}{2}} & e^{-\frac{\delta}{2}} \gamma_{5} \\
e^{-\frac{\delta}{2}} \gamma_{5} & -e^{\frac{\delta}{2}}
\end{array}\right) \quad \text { where } \sinh \delta=\frac{g_{1} \sigma_{0}}{m_{0}} .
$$

Note that the mixing angle $\delta$ depends on $\sigma_{0}$. This is in contrast with the naive case. The physical masses are obtained as

$$
M_{ \pm}=\mp g_{2} \sigma_{0}+\sqrt{\left(g_{1} \sigma_{0}\right)^{2}+m_{0}^{2}} .
$$

To get the physical pion couplings, we apply the unitary transformation also to $\Pi$, which then leads to the axial charges,

$$
\begin{aligned}
g_{A N N} & =\tanh \delta-\frac{g_{4} \sigma_{0}{ }^{2} e^{\delta}-g_{5} \sigma_{0}{ }^{2} e^{-\delta}}{2 \cosh \delta}+\frac{2 \sigma_{0} g_{3}}{\cosh \delta}, \\
g_{A N N^{*}} & =\frac{2-\sigma_{0}{ }^{2}\left(g_{4}+g_{5}\right)}{2 \cosh \delta}-2 \sigma_{0} g_{3} \tanh \delta \\
g_{A N^{*} N^{*}} & =-\tanh \delta-\frac{g_{4} \sigma_{0}{ }^{2} e^{-\delta}-g_{5} \sigma_{0}{ }^{2} e^{\delta}}{2 \cosh \delta}-\frac{2 \sigma_{0} g_{3}}{\cosh \delta} .
\end{aligned}
$$

Unfortunately, we have six undetermined parameters in this extension while there are only four experimental inputs,

$$
\begin{aligned}
& M_{-}=1.535 \mathrm{GeV} ; \quad M_{+}=0.939 \mathrm{GeV}, \\
& g_{A N N}=1.26 ; \quad g_{A N N^{*}}=0.217 .
\end{aligned}
$$

One more constraint can be imposed by noting that $g_{A N N}$ and $g_{A N^{*} N^{*}}$ are differed only by the quadratic terms in meson fields. Since the quadratic and linear terms in meson fields were introduced in a way to improve the linear sigma model, the difference should be small. Otherwise, the truncation in number of meson fields can not be justified. This means that $g_{A N N}$ can be assumed to be similar or even equal in magnitude with $g_{A N^{*} N^{*}}$. Also, as in the 
original DK model, we expect the sign of $g_{A N^{*} N^{*}}$ to be the opposite of $g_{A N N}$. In Section IV, we will present the results with the condition, $g_{A N N}=-g_{A N^{*} N^{*}}$, which yields $g_{4}=g_{5}$. The reliability of this constraint can be checked by allowing a small deviation $\epsilon$ such that

$$
g_{A N N}=-g_{A N^{*} N^{*}}+\epsilon
$$

One more freedom in our model will be fixed by taking $m_{0}$ as an adjustable parameter for our predictions. In principle, $m_{0}$ should be determined for example by lattice calculations. In the DK model, $g_{A N N}$ is proportional only to $\tanh \delta$. Since their $m_{0}$ is fixed to a small value, $g_{A N N}$ is a very smooth function of $\sigma_{0}$. Practically one can start to see a noticeable reduction of $g_{A N N}$ only when $\sigma_{0}$ is as small as $20 \mathrm{MeV}$. Therefore, the so called " $g_{A N N}$ quenching" at the normal nuclear density can not be observed within their model. However, in our extension, $m_{0}$ is not necessarily fixed to a small value and it is possible that part of the $g_{A N N}$ quenching can be driven by this mechanism.

\section{RESULTS AND DISCUSSIONS}

In the last two sections, we have improved the "naive" and "mirror" models for the nucleon parity doublets. The additional chiral symmetric terms are introduced so that the naive and mirror models can be consistent with phenomenology in free space. One important consequence of those terms is that physical quantities such as masses or couplings are functions of $\sigma_{0}$. As $\sigma_{0}$ is expected to be changed in the nuclear medium, our formalism could reveal interesting density dependence of those quantities.

To make realistic predictions, we calculate $\sigma_{0}$ in Hartree approximation as a function of the nuclear density. A similar calculation for the mirror model has been performed by Hatsuda and Prakash [15]. Since we use the pseudovector coupling for pion, Fock exchange terms are not expected to to be large [11]. As the nucleus in the ground state does not contain $N^{*}$, there is no Fermi energy for $N^{*}$ and the energy density in Hartree approximation takes the form,

$$
\mathcal{E}=\lambda\left(\sigma_{0}^{2}-f_{\pi}^{2}\right)^{2}+\mathcal{E}_{v}^{+}+\mathcal{E}_{v}^{-}+4 \int_{0}^{k_{F}} \frac{d^{3} k}{(2 \pi)^{3}} \sqrt{\mathbf{k}^{2}+M_{+}^{* 2}},
$$

where $M_{+}^{*}$ denotes the positive-parity nucleon mass in the medium. The constant $\lambda$ in front of the meson energy is related with the mass of the $\sigma$ meson via $\lambda=m_{\sigma}^{2} / 8 f_{\pi}^{2}$. In our calculation, we take $m_{\sigma}=600 \mathrm{MeV}$ as in Ref. [15]. This $m_{\sigma}$ is also supported by recent analysis of $\pi \pi$-scattering phase shift [16]. The factor 4 in the last term indicates that we are considering the symmetric nuclear matter. We do not include the $\omega$ meson term interacting with nucleons simply because it does not participate in determining $\sigma_{0}$ in matter. The vacuum contributions from the positive-(negative-)parity nucleon to the energy density, $\mathcal{E}_{v}^{+}$ $\left(\mathcal{E}_{v}^{-}\right)$, diverge. Suitable counter terms [10] are introduced to obtain

$$
\begin{aligned}
\mathcal{E}_{v}^{ \pm} & =-\frac{1}{4 \pi^{2}}\left[M_{ \pm}^{* 4} \ln \frac{M_{ \pm}^{*}}{M_{ \pm}}-M_{ \pm}^{3}\left(M_{ \pm}^{*}-M_{ \pm}\right)-\frac{7}{2}\left(M_{ \pm}^{*}-M_{ \pm}\right)^{2} M_{ \pm}^{2}\right. \\
& \left.-\frac{13}{3} M_{ \pm}\left(M_{ \pm}^{*}-M_{ \pm}\right)^{3}-\frac{25}{12}\left(M_{ \pm}^{*}-M_{ \pm}\right)^{4}\right]
\end{aligned}
$$


Now we minimize the energy density to obtain $\sigma_{0}$ at a certain density which is then used to determine the values of $M_{ \pm}^{*}$ and the axial charges, $g_{A N N}$ and $g_{A N N^{*}}$.

Figure 1 (a) shows our results from Hartree calculation for $\sigma_{0}$ as a function of density. We consider up to twice of the normal nuclear density, a range where this effective model approach is reliable. The two models clearly predict $\sigma_{0}$ to decrease as the density increases. The curve for the mirror case is obtained by using $m_{0}=0.5 \mathrm{GeV}$. Larger $m_{0}$ leads to slow decreasing rate for $\sigma_{0}$ but dependence on $m_{0}$ is not strong. The slope of $\sigma_{0}$ at $\rho=0$ is special as it is related to $\pi N$-sigma term, $\Sigma_{\pi N}$, via $d \sigma_{0}^{2} /\left.d \rho\right|_{\rho=0}=-\Sigma_{\pi N} / m_{\pi}^{2}$. The two curves in Fig. 1 (a) yield $\Sigma_{\pi N} \sim 100 \mathrm{MeV}(\sim 80 \mathrm{MeV})$ for the naive case (the mirror case) which is larger than the typical value $\Sigma_{\pi N} \sim 45 \mathrm{MeV}$. The slope at the zero density however is somewhat sensitive to $m_{0}$. For $m_{0}=700 \mathrm{MeV}$, we find $\Sigma_{\pi N} \sim 60 \mathrm{MeV}$ which is close to the typical value. In this regards, our model with larger $m_{0}$ seems to be favored. Larger $m_{0}$ is also suggested by lattice calculations [6].

Using the density dependence of $\sigma_{0}$ as an input, we calculate the doublet masses which are shown in Fig. 1 (b). In the mirror case, we have used $m_{0}=0.5 \mathrm{GeV}$. We have also tried other values of $m_{0}$ and found that the changes are at most $10 \mathrm{MeV}$ at $\rho_{0}$. Also shown is the $\eta$-meson mass (dot-dashed line) in vacuum. The two models predict $M_{ \pm}^{*}$ to decrease as the density increases, seemingly consistent with the Brown-Rho scaling [13]. Since the decreasing rate for $M_{+}^{*}$ is different from that of $M_{-}^{*}$, we find that $M_{-}^{*}-M_{+}^{*}$ is also getting smaller as the density increases, indicating that $N^{*}$ cannot decay to $\eta N$ in the medium if the mass of $\eta$ is assumed not to change in the medium. This feature is insensitive to $m_{0}$.

It is interesting to note that our results support the recent observation [17] where $\eta$ productions from nuclei are found to be scaled with the nuclear surface area. In Ref. [17], the scaling is explained by strong nuclear absorptions of $\eta$ produced inside a nucleus. Here, our results provide an alternative explanation for the scaling. According to our models, $N^{*} \rightarrow \eta N$ decay is possible only at low density region below $\rho / \rho_{0} \sim 0.3$. Therefore, $\eta$ is produced only at the surface of the nucleus and thus the experimentally observed scaling can be explained.

We now turn to results for the axial charges. As discussed above, we here present the results with the condition $g_{A N N}=-g_{A N^{*} N^{*}}$ and discuss the reliability. In Figure 2 (a), the nucleon axial charge, $g_{A N N}$, is shown to decrease as $\rho$ increases. The quenching in the naive case is basically what Akhmedov found in his modified sigma model [8]. At $\rho=\rho_{0}$, $g_{A N N}$ is quenched by $8 \%$. For the mirror case, the quenching rate depends strongly on the choice of $m_{0}$. The axial charge decreases more rapidly for larger $m_{0}$. This trend is basically unchanged when we allow nonzero deviation $\epsilon$ defined in Eq. (22). For example, even with a very large value $\epsilon= \pm 0.5, g_{A N N}$ is changed only by $2 \%$ at $\rho_{0}$. Therefore, the mirror model provides another interesting mechanism for the quenching of the axial charge in medium. When $\sigma_{0}$ decreases towards the chiral restoration $\sigma_{0} \rightarrow 0, g_{A N N}$ approaches to 1 in the naive case while in the mirror case it approaches to zero, because these values are determined by their chiral assignment.

More clear distinction between the two assignments can be seen from the off-diagonal axial charge, $g_{A N N^{*}}$. As shown in Fig. 2 (b), the two models predict opposite trends for $g_{A N N^{*}}$ as the density increases. Starting from $0.217, g_{A N N^{*}}$ in the naive case decreases while its value in the mirror model increases. The increasing rate in the mirror case strongly depends on $m_{0}$. Small $m_{0}$ usually tends to flatten the curve. Specifically, the result with 
$m_{0}=0.3 \mathrm{GeV}$ shows that $g_{A N N^{*}}$ is 0.294 at $\rho_{0}$. Corresponding result from the naive model is 0.15, about a factor of 2 smaller. As is clear from Fig. 2 (b), the gap is much bigger for larger $m_{0}$. We have also checked the sensitivity to $\epsilon$. Depending on its sign, its effect appears with either rapid or slow increasing rate. For example, $\epsilon= \pm 0.5$ changes $g_{A N N^{*}}$ at $\rho_{0}$ by at most $\pm 20 \%$. Therefore, the qualitative aspect of our result is unchanged by the uncertainty of the model. Unlike the diagonal axial charge $g_{A N N}$, the off-diagonal $g_{A N N^{*}}$ in the naive case goes to zero when the chiral symmetry is restored, while in the mirror case $g_{A N N^{*}}$ goes to 1 . This implies that $N^{*}$ is the chiral partner of $N$ [7] .

As $g_{A N N^{*}}$ is related to the pseudovector coupling, $f_{\pi N N^{*}}$, via $2 f_{\pi} f_{\pi N N^{*}}=g_{A N N^{*}}$, the two models predict either suppression or enhancement of $\pi N$ decay of $N^{*}$ in the medium. Effects from the Pauli blocking are not expected to be large as the emitting nucleon has momentum much larger than the Fermi momentum. Since the decay of $N^{*}$ to $\eta N$ will be strongly suppressed (recall the discussion above), we expect that the main mesonic decay mode of $N^{*}$ in nuclei would be $\pi N$. Whether that partial width increases or decreases could be used in determining the realistic assignment of chiral symmetry.

To summarize, we have minimally extended the naive and mirror models for the nucleon parity-doublet by introducing nonlinear terms allowed by respective assignment of chiral symmetry. A unique description of the pion coupling in terms of the pseudovector scheme has been achieved by the nonlinear transformations. We have investigated how the two assignments of chiral symmetry can be differed in our extension by performing Hartree calculation of $\sigma_{0}$ in the nuclear matter. Using the density dependence of $\sigma_{0}$, we have calculated the doublet masses and the axial charges in the medium. We have found that the mass difference, $M_{-}^{*}-M_{+}^{*}$, decreases as the density increases. Therefore, $N^{*}$ cannot decay to $\eta N$ in the medium, agreeing with recent experimental observation [17]. Also both the models predict that the axial charge, $g_{A N N}$, is quenched in the medium. Most remarkable result in our work is that the two models are clearly distinguished by the density dependence of the off-diagonal axial charge, $g_{A N N^{*}}$. Therefore, the realistic assignment of chiral symmetry might be determined by studying $\pi N$ and $\eta N$ decays of $N^{*}$ in medium.

\section{ACKNOWLEDGMENTS}

This work is supported in part by the Grant-in-Aid for scientific research (C) (2) 08640356 and (A) (1) 08304024 of the Ministry of Education, Science, Sports and Culture of Japan. The work of H. Kim and D. Jido is supported by Research Fellowships of the Japan Society for the Promotion of Science. 


\section{REFERENCES}

[1] B. Krushce et al., Phys. Rev. Lett. 74 (1995) 3736; B. Schoch et al., Acta Phys. Polonica 24 (1993) 1765.

[2] M. Benmerrouche and N. C. Mukhopadhyay, Phys. Rev. Lett. 67 (1991) 1070; Z. Li, Phys. Rev. D 52 (1995) 4961; N. Kaiser, P. B. Siegel, and W. Weiss, Phys. Lett. B 362 (1995) 23; Ch. Sauermann, B. L. Friman, and W. Nörenberg, Phys. Lett. B 341 (1995) 261; M. Benmerrouche, N. C. Mukhopadhyay, and J. F. Zhang, Phys. Rev. D 51 (1995) 3237.

[3] S. H. Lee and H. Kim, Nucl. Phys. A 612, 418 (1997); H. Kim and S. H. Lee, Phys. Rev. D. 56, 4278 (1997).

[4] D. Jido, M. Oka, and A. Hosaka, Phys. Rev. Lett. 80, 448 (1998); D. Jido, N. Kodama and M. Oka, Phys. Rev. D 54, 4532 (1996).

[5] C. DeTar and T. Kunihiro, Phys. Rev. D. 39, 2805 (1989).

[6] S. Gottlieb, W. Liu, D. Toussaint, R. L. Renkin, and R. L. Sugar, Phys. Rev. Lett. 59, 1881 (1987); C. E. DeTar and J. B. Kogut, Phys. Rev. Lett. 59, 399 (1987).

[7] D. Jido, Y. Nemoto, M. Oka, and A. Hosaka, Los Alamos Preprint hep-ph/9805306

[8] E. Kh. Akhmedov, Nucl. Phys. A 500, 596 (1989).

[9] B. W. Lee, Chiral Dynamics (Gordon and Breach, NY, 1972).

[10] B. D. Serot and J. D. Walecka, Advances in Nuclear Physics, edited by J.W. Negele and E. Vogt (Plenum, New York, 1986), Vol. 16.

[11] C. J. Horowitz and B. D. Serot, Nucl. Phys. A 399, 529 (1983).

[12] S. Weinberg, Phys. Rev. 166, 1568 (1968).

[13] G. E. Brown and M. Rho, Phys. Rev. Lett. 66, 2720 (1991).

[14] The term "mirror fermion" is introduced in the paper; J. Maalampi and M. Roos, Phys. Rep. 186,53 (1990)

[15] T. Hatsuda and M. Prakash, Phys. Lett. B 224 (1989) 11.

[16] M. Ishida, Ph.D thesis, University of Tokyo (1997); S. Ishida, T. Ishida, M. Ishida, K.Takamatsu, T. Tsuru, Prog. Theor. Phys. 98,1005 (1997).

[17] M. Röbig-Landau et al., Phys. Lett. B 373 (1996) 45. 


\section{FIGURES}

FIG. 1. Figure (a) shows the density dependence of $\sigma_{0}$. The solid line is for the naive case and the dashed line is for the mirror case. Figure (b) shows our predictions for $M_{-}^{*}, M_{+}^{*}$ and the mass difference $M_{-}^{*}-M_{+}^{*}$ as density increases. The solid lines (dashed lines) are for the naive case (mirror case). For the mirror case, $m_{0}=0.5 \mathrm{GeV}$ is used. The two curves for $M_{-}^{*}-M_{+}^{*}$ are almost indistinguishable. Also shown with the dot-dashed line is the mass of $\eta$ in free space.

FIG. 2. Our prediction for axial charges. The solid lines (dashed lines) are the results from the naive case (mirror case). Figure (a) is for the nucleon axial charge and (b) is for the off-diagonal axial charge. The results from the mirror case are shown for $m_{0}=0.3,0.5$ and $0.7 \mathrm{GeV}$ as indicated. 


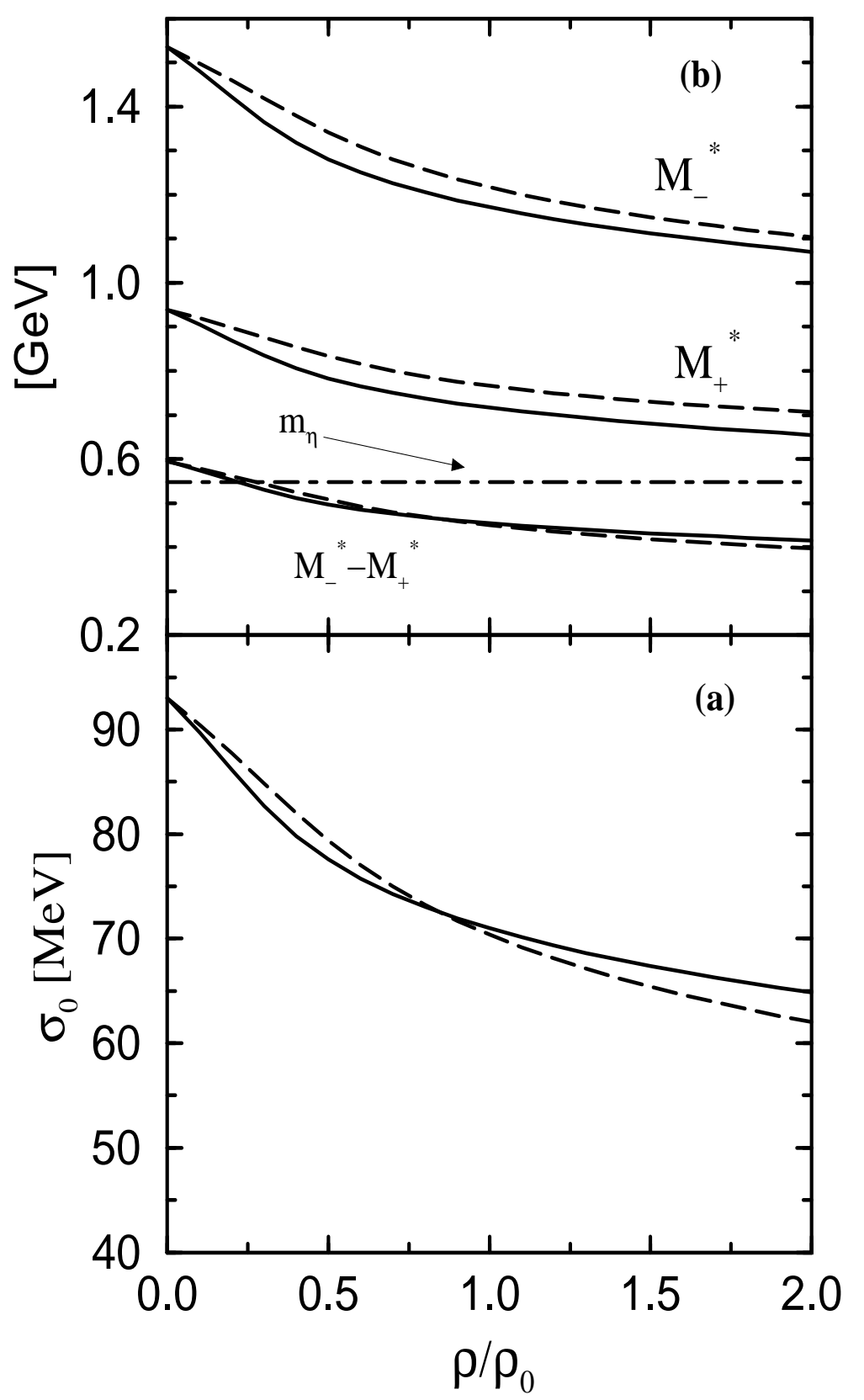

Figure 1 


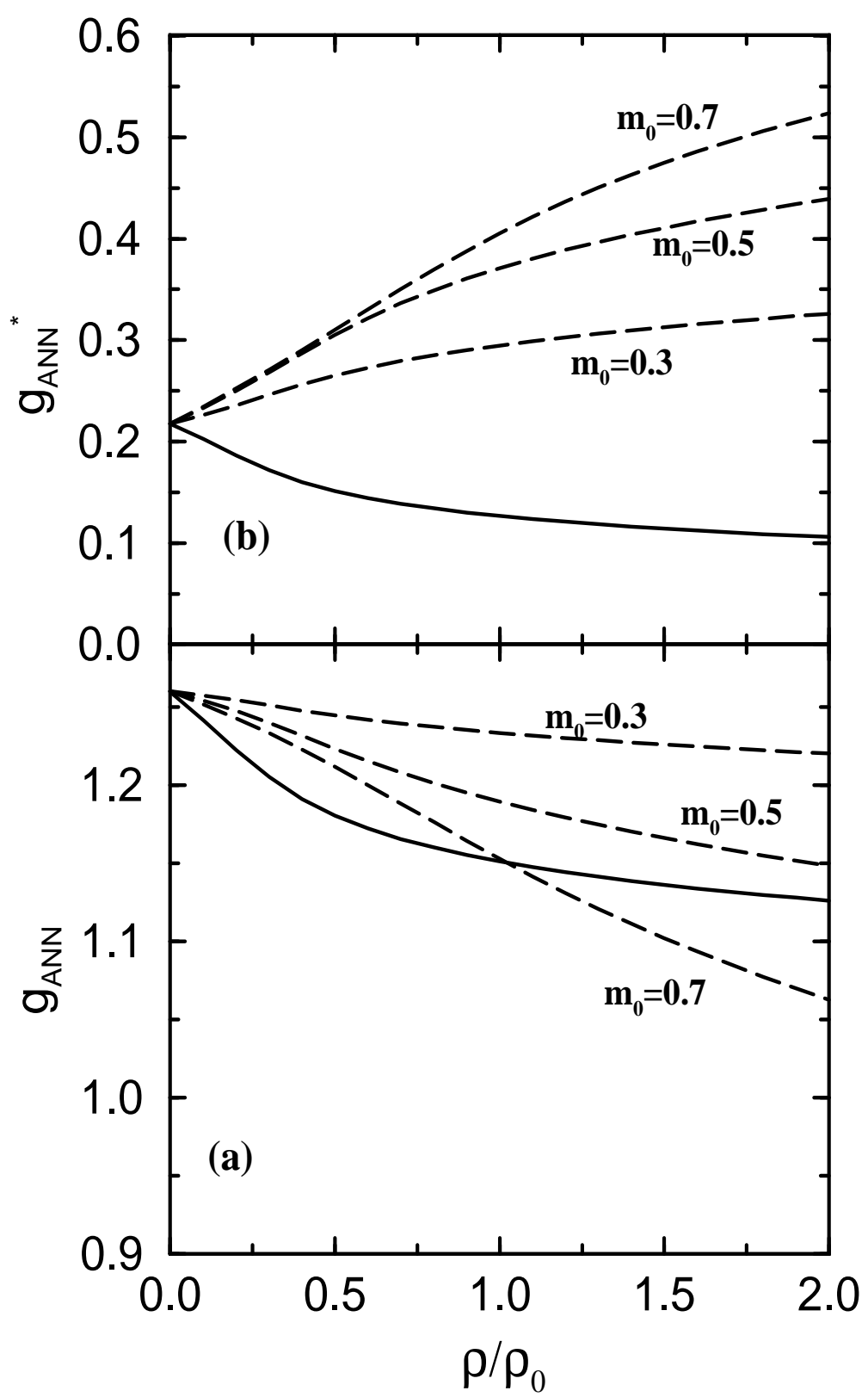

Figure 2 